\title{
Metformin in non-alcoholic fatty liver disease: A systematic review and meta-analysis
}

\author{
YAN LI ${ }^{1}$, LEI LIU ${ }^{2}$, BIN WANG $^{1}$, JUN WANG $^{1}$ and DONGFENG CHEN ${ }^{1}$ \\ ${ }^{1}$ Department of Gastroenterology, Institute of Surgery Research, Daping Hospital, Third Military Medical University, \\ Chongqing 400042; ${ }^{2}$ Biowave Center and Department of Natural Medicinal Chemistry, College of Pharmacy, \\ Third Military Medical University, Chongqing 400038, P.R. China
}

Received July 23, 2012; Accepted September 18, 2012

DOI: $10.3892 /$ br.2012.18

\begin{abstract}
Non-alcoholic fatty liver disease (NAFLD) related to insulin resistance (IR) is a growing global health concern. Recent studies have indicated that metformin could improve IR and may be beneficial in the treatment of NAFLD. This study aimed to assess the beneficial or harmful effects of metformin in NAFLD. We searched Medline and four other databases during April 2012. Selection criteria were randomized clinical trials comparing metformin with placebo or other interventions for treating NAFLD patients. The primary outcome was histological response. The secondary outcomes included alanine aminotransferase (ALT), aspartate aminotransferase (AST), homeostasis model assessment of IR (HOMA-IR), body mass index (BMI) and adverse events. Dichotomous data were reported as odds ratio (OR), while continuous data were calculated as the mean difference (MD), both with $95 \%$ confidence intervals (CI). Random and fixed effects meta-analyses were performed. Nine studies were included, involving 417 participants, and conducted for a time period ranging from 4 to 12 months. In the treated participants, improvements were observed in ALT (MD, -8.12 U/1; P=0.03), AST (MD, -4.52 U/1; P=0.04), HOMA-IR (MD, -0.61; $\mathrm{P}=0.005$ ) and BMI (MD, $\left.-0.82 \mathrm{~kg} / \mathrm{m}^{2} ; \mathrm{P}=0.04\right)$, but not in histological response: steatosis $(\mathrm{P}=0.66)$, inflammation $(\mathrm{P}=0.91)$, hepatocellular ballooning $(\mathrm{P}=0.25)$ and fibrosis $(\mathrm{P}=0.90)$. Sub-analysis of
\end{abstract}

Correspondence to: Professor Dongfeng Chen or Dr Jun Wang, Department of Gastroenterology, Institute of Surgery Research, Daping Hospital, Third Military Medical University, 10 Changjiang Zhilu, Da Ping, Chongqing 400042, P.R. China

E-mail: chendf1981@163.com

E-mail: wangjunxhk@163.com

Abbreviations: ALT, alanine aminotransferase; AST, aspartate aminotransferase; BMI, body mass index; CI, confidence intervals; HOMA-IR, homeostasis model assessment of insulin resistance; IR, insulin resistance; MD, mean difference; NAFLD, non-alcoholic fatty liver disease; NASH, non-alcoholic steatohepatitis; RCT, randomized clinical trial; SD, standard deviation; OR, odds ratio; US, ultrasonography

Key words: metformin, metabolic liver disease, therapy non-alcoholic fatty steatohepatitis showed that metformin failed to improve any pooled outcome. Adverse events were poorly reported. Current information indicates that metformin improves liver function, HOMA-IR and BMI to some extent, but not histological response in NAFLD patients. This finding could serve as a stimulus for future studies investigating issues such as dose-responsiveness, safety and patient tolerance to metformin therapy.

\section{Introduction}

Non-alcoholic fatty liver disease (NAFLD) is a burgeoning health problem that affects $1 / 3$ of the adult population and an increasing number of children in developed countries $(1,2)$. NAFLD includes a wide spectrum of histologic abnormalities ranging from hepatic steatosis (3) to non-alcoholic steatohepatitis (NASH) that may progress to cirrhosis, and subsequent end-stage liver disease and hepatocellular carcinoma $(4,5)$. NAFLD is characterized by fat accumulation in the liver in patients with no statistically significant alcohol consumption, and is particularly associated with metabolic syndrome comprising hypertension, insulin resistance (IR), obesity and dyslipidaemia (6). NAFLD has been shown to be strongly and independently associated with an increased risk of type 2 diabetes and cardiovascular disease $(7,8)$.

The pathogenesis of NAFLD is complex and not fully elucidated. The classical supporting theory is the 'two-hit' hypothesis $(9,10)$, whereby IR leads to hepatic steatosis (first hit), while the steatosis subsequently sensitizes the liver to a variety of metabolic injuries (second hit) leading to necroinflammation and fibrosis. Since IR is crucial in the pathogenesis of NAFLD, insulin-sensitizing drugs, such as metformin may offer a therapeutic benefit. Metformin probably interrupts mitochondrial oxidative processes, resulting in a reduced ATP/ AMP ratio and subsequently in the activation of AMP-activated protein kinase, a major cell regulator of lipid and glucose metabolism $(11,12)$. The activation of AMP-activated protein kinase in the liver stimulates $\beta$-oxidation of fatty acids and inhibits de novo synthesis, thus potentially leading to reduced liver steatosis (13).

At present, the ideal treatment for NAFLD has yet to be identified. Current treatments, such as lifestyle modification and weight loss, are often difficult to achieve and hard 
to maintain (14). Therefore, new therapeutic approaches for the management of NAFLD are required. Metformin is non-hepatotoxic and cost-effective, therefore, it is more suitable for long-term treatment (15). Thus, a number of clinical trials have evaluated the use of metformin in the treatment of NAFLD $(16,17)$. However, the mixed results, heterogeneous therapeutic approaches and small number of patients have limited its widespread use in clinical practice. A recent meta-analysis of randomized control trials (RCT) including sub-analysis of the efficacy of metformin on histological and biochemical outcomes in biopsy-proven NASH, suggested that metformin is not an effective treatment for NASH (17). The authors explained the strict inclusion criteria with the histological definition of NAFLD or NASH, while only three RCTs were included as barriers to the meta-analysis with regards to the effect of metformin on NAFLD. However, several studies, including more recent RCT data, and an additional review have shown that metformin may improve metabolic variables in NAFLD patients, especially in patients meeting the diagnostic criteria of metabolic syndrome (18). Therefore, the aim of this systematic review and meta-analysis was to further assess the beneficial and harmful effects of metformin on NAFLD.

\section{Materials and methods}

Search strategy. A systematic computer-assisted search was conducted by two independent investigators (Y.L. and L.L.) with disagreements resolved by mutual discussion. Databases searched during April 2012 included Medline, Cochrane the Central Register of Controlled Trials, the Cochrane Database of Systematic Reviews, Embase, Science Citation Index Expanded and The Chinese Biomedical Database. The meeting proceedings (American Gastroenterological Association/American Association for the Study of Liver Diseases/Digestive Disease Week meeting abstracts/European Association for the Study of the Liver) and reference lists of reviews were searched manually for additional relevant studies. The authors of locally published and unpublished studies were contacted for thoroughness. Search terms included 'Metformin', 'Biguanides', 'Glucophag', 'NAFLD', 'NASH', 'nonalcoholic fatty liver disease', 'nonalcoholic steatohepatitis', 'liver fat', 'fatty liver', 'steatosis', 'AST', 'ALT', 'aminotransferase', 'liver enzymes' and 'trial'.

Inclusion and exclusion criteria. Two investigators (Y.L. and D.C.) determined the inclusion and exclusion criteria, and reviewed the titles and abstracts of the studies identified. Inclusion criteria were: i) RCTs using metformin in patients with NAFLD or NASH; ii) NAFLD or NASH diagnosed by histology or suggestive imaging findings (ultrasound, computed tomography, magnetic resonance imaging) with abnormal aminotransferase; iii) comparators could be placebo, no intervention or other intervention and iv) adult patients of any gender or ethnic origin (age, $\geq 18$ years). Exclusion criteria were: i) non-human studies or non-randomized trials; ii) participants with addressed alcoholic, drug-induced, total parenteral nutrition-induced, viral or genetic causes of liver injury; iii) combination of metformin and other therapeutic approaches (e.g., thiazolidinediones and antioxidants) and iv) letters/case reports or studies enrolling $<10$ subjects, or manuscripts without adequate data or reviews. There was no restriction on languages.

Outcome measures. The primary outcome was a change in histological response quantified by needle biopsy and histological assessment, proton magnetic resonance spectroscopy (1H MRS), or inferred by ultrasonography (US). The secondary outcomes included alanine aminotransferase (ALT), aspartate aminotransferase (AST), insulin sensitivity (measured by homeostasis model assessment of IR 'HOMA-IR') and body mass index (BMI). Incident adverse events were also evaluated.

Data extraction. Two investigators (Y.L. and L.L.) independently assessed the selected studies. Y.L. abstracted data and then L.L. checked the data extraction. The agreement in data extraction measured by a $\kappa$ statistic and discrepancies were resolved by consensus. The data extracted included: i) study: date, location and funding of the trial, length of follow-up, use of intention-to-treat analyses and the publication status; ii) patients: number, inclusion and exclusion criteria, mean (or median) age and gender ratio; iii) treatment: dose, duration and mode of administration of various metformin and/or of additional comparators and v) outcome: histological response, ALT, AST, BMI, HOMA-IR and adverse effects.

Missing data. In the cases where the information of interest was not presented in the published studies, the investigators contacted the corresponding authors to obtain additional information. For incomplete or missing dichotomous data, sensitivity analysis was performed by excluding the studies that appeared as outliers in the forest plots. For missing standard deviations (SD) of the mean change in continuous data, and where the P-value was provided for the two groups, SD was calculated by converting the P-value into a t-value with appropriate degrees of freedom (19). Studies in which participants dropped out, thereby presenting these percentages, were not excluded.

Methodological quality assessment. Methodological quality was assessed by two investigators (Y.L. and J.W.) using the Cochrane Risk of Bias Tool with a potential risk of bias of high, low or unclear (19). Quality assessment was based on the following domains: i) sequence generation; ii) allocation concealment; iii) blinding of participants, personnel and outcome assessors; iv) completeness of outcome data; v) unbiased outcome reporting and vi) source of funding. If all six domains were well-described, the studies were categorized as high quality, otherwise as low quality.

Data analysis. The analyses were conducted using the Review Manager (RevMan Version 5.1.6 Copenhagen: The Cochrane Collaboration 2012). The primary outcome (histological response) was assessed as a dichotomous variable (presented as OR with 95\% CI). The secondary outcomes (ALT, AST, HOMA-IR and BMI) were presented as continuous variables (presented as MD and 95\% CI). Five analyses were carried out to compare the effect of i) metformin vs. control on liver histological response, ii) metformin vs. control on ALT change and iii) metformin vs. control on AST change, iv) metformin vs. control on BMI change and v) metformin vs. 


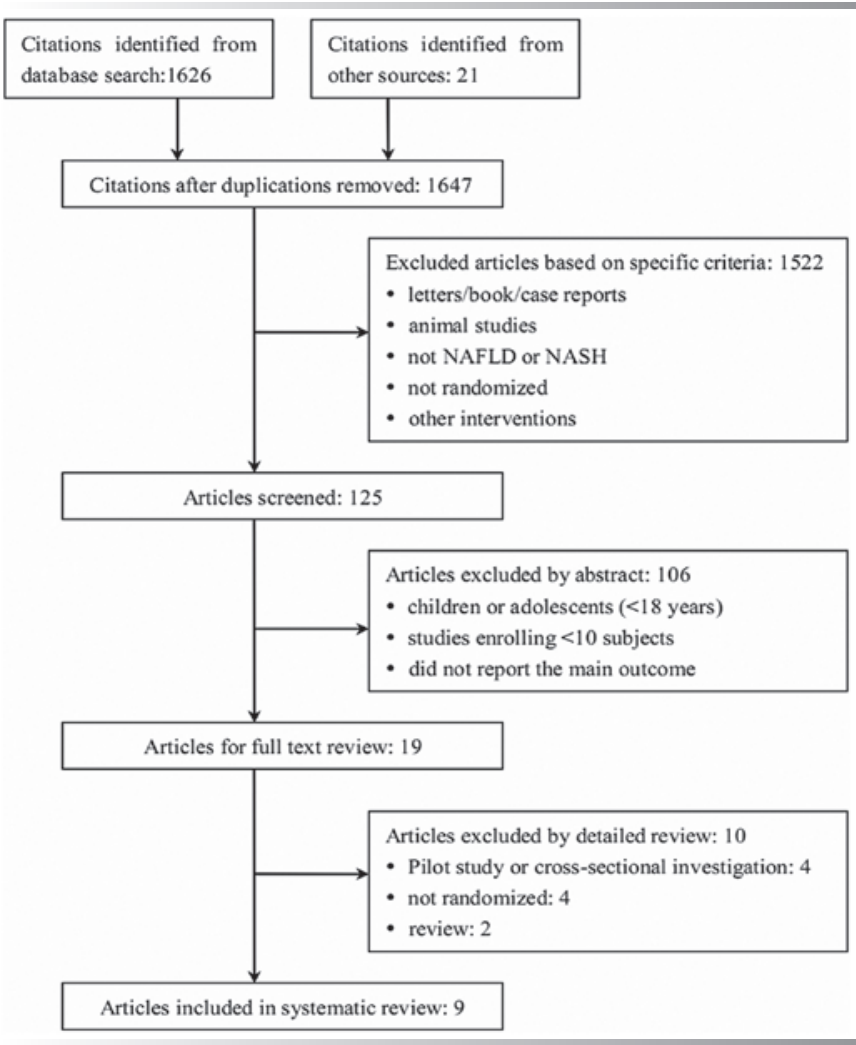

Figure 1. Flowchart of the literature search and selection process is shown. NAFLD, non-alcoholic fatty liver disease; NASH, non-alcoholic steatohepatitis.

control on HOMA-IR change. Random effect models were used in most meta-analyses, while statistical heterogeneity was evaluated using Cochran's $\mathrm{Q}$ statistic $(\mathrm{P}<0.10)$ and the $\mathrm{I}^{2}$ statistic. Where Cochran's Q statistic $(\mathrm{P}>0.10)$ or an $\mathrm{I}^{2}$ statistic was $>50 \%$, the summary meta-analysis was abandoned and potential sources were examined using stratified analyses. Subgroup analysis was carried out for two groups: i) diabetics and non-diabetics; ii) NAFLD and NASH population. Sensitivity analysis was performed by excluding lower quality studies from the meta-analysis and assessing the effect on the summary estimate.

\section{Results}

Literature search. A total of 1,647 non-duplicated studies were retrieved from the broad search terms used. After review of the titles and abstracts of these studies, 1,628 were excluded. Nineteen studies underwent full text review and 10 were subsequently excluded for the following reasons: 4 were pilot studies or cross-sectional investigations, 4 were not randomized and 2 were reviews. Therefore, 9 studies were included in this systematic review and meta-analysis (Fig. 1).

Trial characteristics. The trials were published in English between 2004 and 2011 and reported similar inclusion and exclusion criteria (Table I). The nine trials included comprised a total study population of 417 individuals. The dose of metformin ranged from 0.5 to $3 \mathrm{~g} / \mathrm{day}$, while the duration of treatment was 4 months in a sole study, 6 months in four studies and 12 months in the remaining four studies. Three

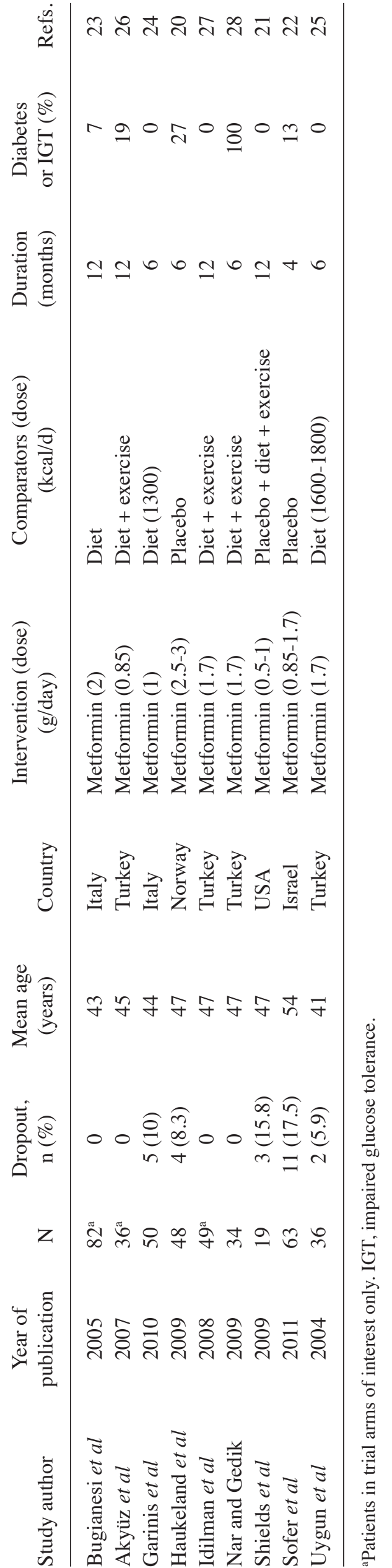


A

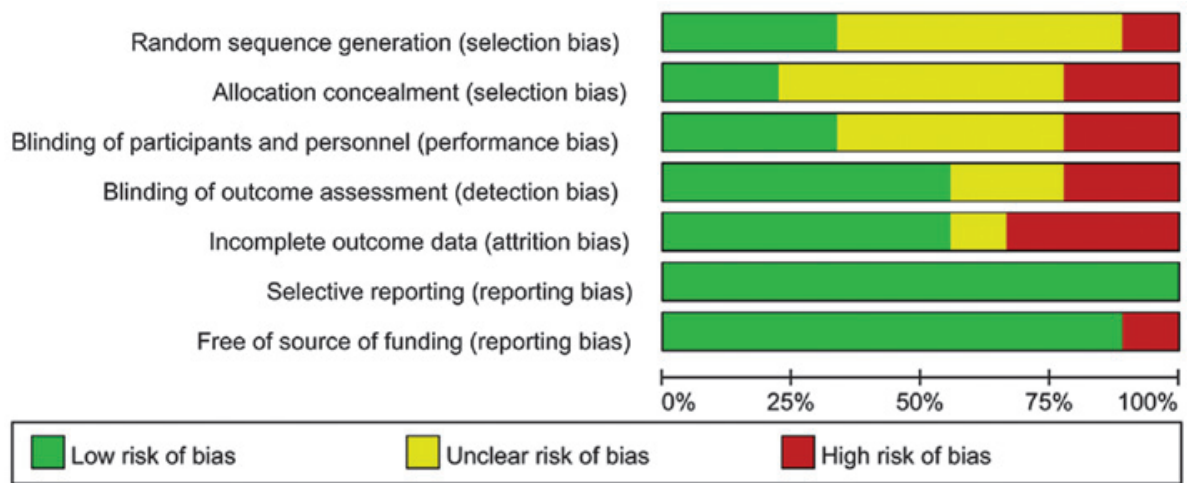

B

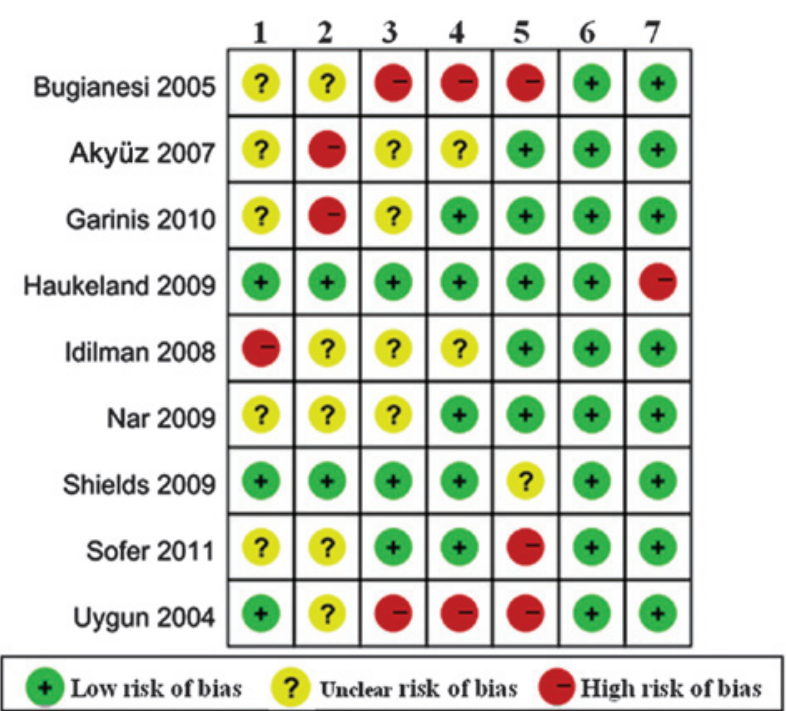

Figure 2. Methodological quality of the studies included in this systematic review is shown. (A) Risk of bias graph for the studies included in this systematic review. (B) Lanes 1, Random sequence generation; 2, allocation concealment; 3, blinding of participants and personnel; 4, blinding of outcome assessment; 5 , incomplete outcome data; 6 , selective reporting; 7 , free of source of funding.

trials were placebo-controlled $(\mathrm{n}=130)(20-22)$, three used diet as the control $(n=168)(23-25)$, while the remaining three trials used diet and exercise as the control $(n=119)(26-28)$. The mean age of the participants ranged from 41 to 54 years.

Methodological quality and risk of bias. The consensus between two investigators regarding the study selection and the quality assessment of trials was 0.81 and 0.92 , respectively. The methodological quality and risk of bias of the nine included studies are shown in Fig. 2. Overall, three studies $(20,21,25)$ had a low risk of bias in random sequence generation and two studies $(20,21)$ had a low risk of bias in allocation concealment. Blinding of outcome assessment was performed in five studies $(20-22,24,28)$ and only one study was funded by a Health Authority (20).

Outcomes reporting. The primary outcome was reported separately (Table II). Two studies $(24,28)$ provided the data of the liver fatty changes diagnosed by US. Four studies $(20,21,25,27)$ reported the number of patients that improved following diagnosis by liver biopsy. The secondary outcomes were integrated, especially with respect to ALT and AST. Partial data of BMI and HOMA-IR were obtained from the figures provided, only a small proportion of data was not reported.

\section{Primary outcome}

Histological response. Histological response was assessed only in four studies $(20,21,25,27)$, evaluating the scores of steatosis, inflammation, hepatocellular ballooning and fibrosis prior and subsequent to treatment. Since these scores were not continuous variables, we calculated the number of patients with improvement in each histological variable. Participants treated with metformin showed no improvement in histological variables compared to those treated with placebo or diet and exercise. OR was 1.30 (95\% CI, 0.414.08; $\mathrm{I}^{2}=47 \%$; $\mathrm{P}=0.66$ ) for steatosis, 1.08 (95\% CI, 0.29-3.99; $\mathrm{I}^{2}=39 \%$; $\mathrm{P}=0.91$ ) for inflammation, 1.89 (95\% CI, 0.64-5.64; $\mathrm{I}^{2}=37 \% ; \mathrm{P}=0.25$ ) for hepatocellular ballooning, and 0.93 (95\% CI, 0.31-2.83; $\mathrm{I}^{2}=0 \% ; \mathrm{P}=0.90$ ) for fibrosis (Fig. 3). When liver fat [diagnosed by US $(24,28)$ ] data alone were analyzed, metformin did not affect any liver fat $(\mathrm{OR}=0.93 ; 95 \% \mathrm{CI}$, $0.32-2.74 ; \mathrm{I}^{2}=0 \% ; \mathrm{P}=0.89$ ). No improvement was observed in the histological variables in the subgroup analyses of diabetic or non-diabetic patients.

\section{Secondary outcomes}

$A L T$. ALT activity was evaluated in the included studies. A statistically significant reduction was observed in ALT in metformin-treated patients (MD, $-8.12 \mathrm{U} / 1 ; 95 \% \mathrm{CI},-15.41$ to 
Table II. Primary and secondary outcomes of the included studies.

\begin{tabular}{|c|c|c|c|c|c|c|c|c|}
\hline \multirow[b]{2}{*}{ Study author } & \multirow{2}{*}{$\begin{array}{c}\text { Year of } \\
\text { publication }\end{array}$} & \multicolumn{2}{|c|}{ Primary outcome } & \multicolumn{4}{|c|}{ Secondary outcomes } & \multirow[b]{2}{*}{ Refs } \\
\hline & & Population (diagnosis) & Histology & ALT & AST & BMI & HOMA-IR & \\
\hline Bugianesi et al & 2005 & NAFLD (biopsy) & Incomplete data ${ }^{a}$ & Fig. 4 & NR & Yes & Fig. 4 & 23 \\
\hline Akyüz et al & 2007 & NAFLD (US and biopsy) & Incomplete data ${ }^{\mathrm{a}}$ & Yes & Yes & Yes & Yes & 26 \\
\hline Garinis et al & 2010 & NAFLD (US) & Fatty changes data ${ }^{\mathrm{b}}$ & Yes & Yes & Yes & Yes & 24 \\
\hline Haukeland et al & 2009 & NAFLD (biopsy) & Number improved & Fig. 4 & Fig. 4 & Yes & Yes & 20 \\
\hline Idilman et al & 2008 & NASH (biopsy) & Number improved & Yes & Yes & Yes & Yes & 27 \\
\hline Nar and Gedik & 2009 & NAFLD (US) & Fatty changes data ${ }^{\mathrm{b}}$ & Yes & Yes & Yes & Yes & 28 \\
\hline Shields et al & 2009 & NASH (biopsy) & Number improved & Fig. 4 & Fig. 4 & Fig. 4 & Fig. 4 & 21 \\
\hline Sofer et al & 2011 & NAFLD (US) & NR & Yes & Yes & NR & Yes & 22 \\
\hline Uygun et al & 2004 & NASH (US and biopsy) & Number improved & Yes & Yes & Yes & NR & 25 \\
\hline
\end{tabular}

${ }^{a}$ No second liver biopsy data were available in the control group, thus unable to be included in meta-analysis. ${ }^{b}$ Fatty changes were subdivided into three groups: low $(<30 \%)$, moderate $(30-50 \%)$ and high grade $(>50 \%)$. ALT, alanine aminotransferase; AST, aspartate aminotransferase; BMI, body mass index; HOMA-IR, homeostasis model assessment of insulin resistance; NAFLD, non-alcoholic fatty liver disease; NR, not reported; US, ultrasonography.

A

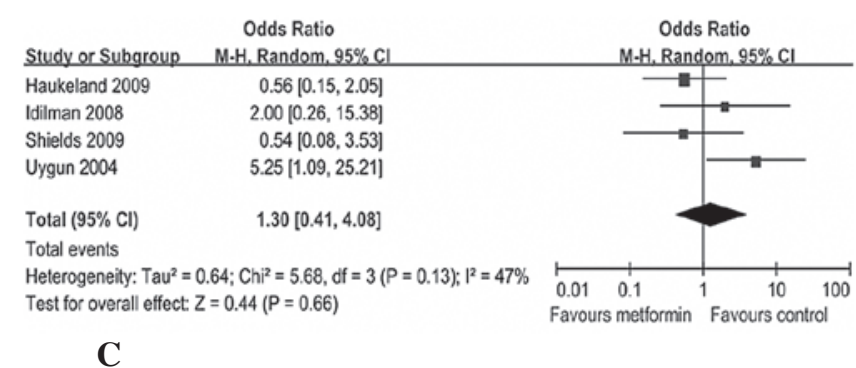

C

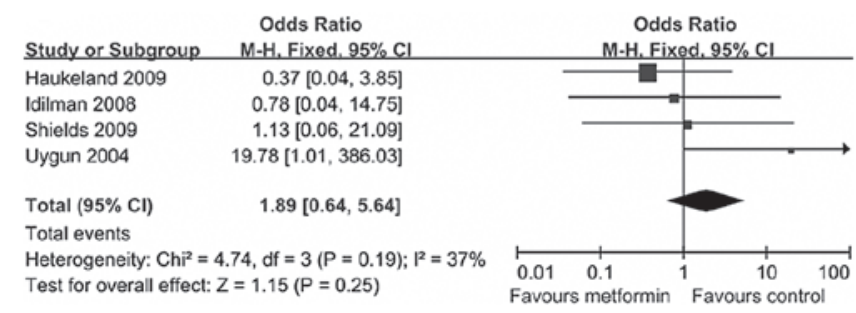

B

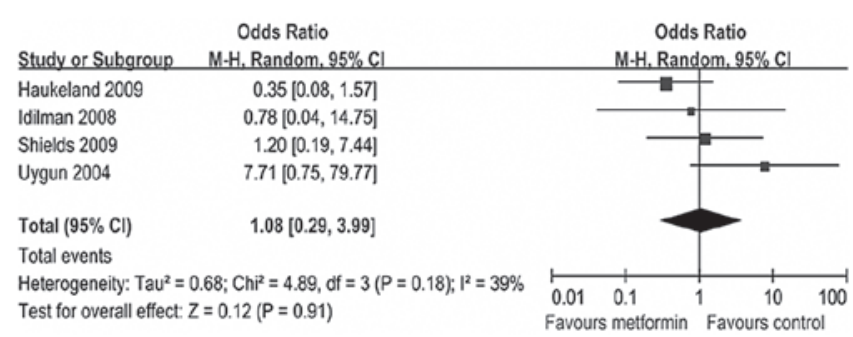

D

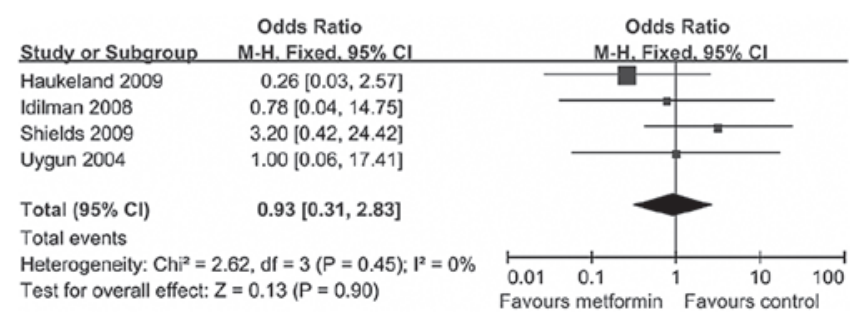

Figure 3. Forest plots of improvement in histological variables are shown. (A) Steatosis; (B) inflammation; (C) hepatocellular ballooning and (D) fibrosis.

$-0.82 \mathrm{U} / \mathrm{l} ; \mathrm{P}=0.03)$ (Fig. 4A). Heterogeneity was high $\left(\mathrm{I}^{2}=69 \%\right)$. Subgroup analysis showed improvement in ALT in NAFLD (MD, -8.44 U/1; $\mathrm{I}^{2}=73 \%$; $\mathrm{P}=0.04$ ), but not in NASH patients (MD, -5.18 U/1; $\mathrm{P}=0.62$ ), although the analysis of the two subgroups demonstrated statistically significant heterogeneity $\left(\mathrm{I}^{2}=73 \%\right)$.

AST. AST was reported in $8 / 9$ studies. Overall, metformin-treated patients showed only a modest, but statistically significant $(\mathrm{P}=0.04)$ decrease in AST $(\mathrm{MD},-4.52 \mathrm{U} / 1$; 95\% CI, -8.91 to $-0.12 \mathrm{U} / 1 ; \mathrm{I}^{2}=63 \%$ ) compared to placebo or diet and exercise (Fig. 4B). In the subgroup analysis, significant differences were observed in the metformin and control groups in NAFLD (MD, $-2.62 \mathrm{U} / 1 ; \mathrm{I}^{2}=0 \% ; \mathrm{P}=0.05$ ), but not in NASH patients (MD, $-6.69 \mathrm{U} / 1 ; \mathrm{P}=0.33$ ), although analysis of the latter subgroup showed a marked heterogeneity $\left(\mathrm{I}^{2}=78 \%\right)$.
HOMA-IR. HOMA-IR activity was reported in eight studies, showing a modest reduction in metformin-treated patients (MD, $-0.61 ; 95 \% \mathrm{CI},-1.04$ to $-0.19 ; \mathrm{I}^{2}=21 \% ; \mathrm{P}=0.005$ ) (Fig. 4C). However, in the subgroup analysis, statistically significant differences were only found in NAFLD (MD, $-0.51 ; \mathrm{I}^{2}=34 \% ; \mathrm{P}=0.04$ ) but not in NASH patients (MD, -1.47 ; $\left.\mathrm{I}^{2}=0 \% ; \mathrm{P}=0.07\right)$.

$B M I$. A noteworthy benefit of metformin therapy vs. the control was observed on BMI (MD, $-0.82 \mathrm{~kg} / \mathrm{m}^{2} ; 95 \%$ CI, -1.61 to $-0.03 \mathrm{~kg} / \mathrm{m}^{2} ; \mathrm{I}^{2}=0 \% ; \mathrm{P}=0.04$ ) (Fig. $4 \mathrm{D}$ ). In the subgroup analysis of NASH, the mean reduction in BMI was modest and statistically not significant (MD, $-0.53 \mathrm{~kg} / \mathrm{m}^{2}, \mathrm{I}^{2}=0 \% ; \mathrm{P}=0.36$ ).

Adverse events. Of the nine studies, only four provided detailed information on gastrointestinal side-effects, such as gas, bloating and mild abdominal pain $(n=17,8.5 \%)$ 
A

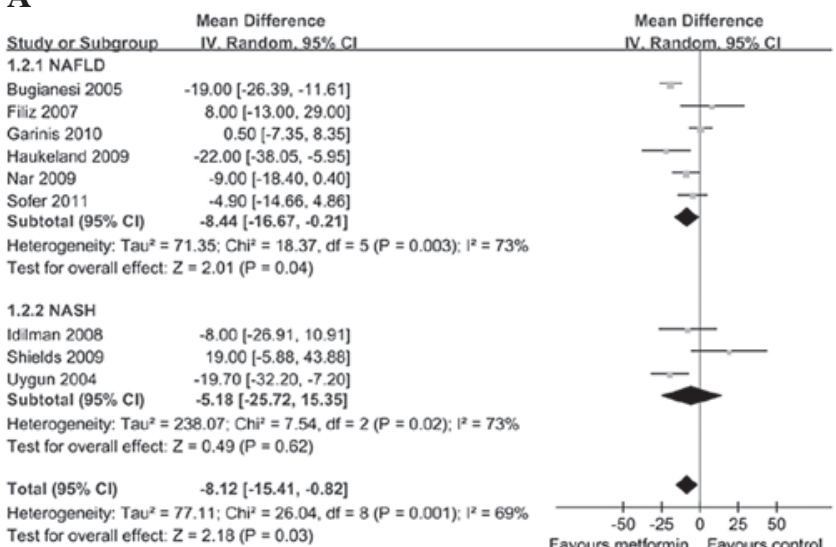

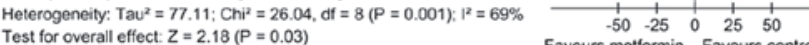

Test for subcrouo differences: $C$ i $i^{2}=0.08$, df $=1(P=0.77) .1^{2}=0 \%$

C

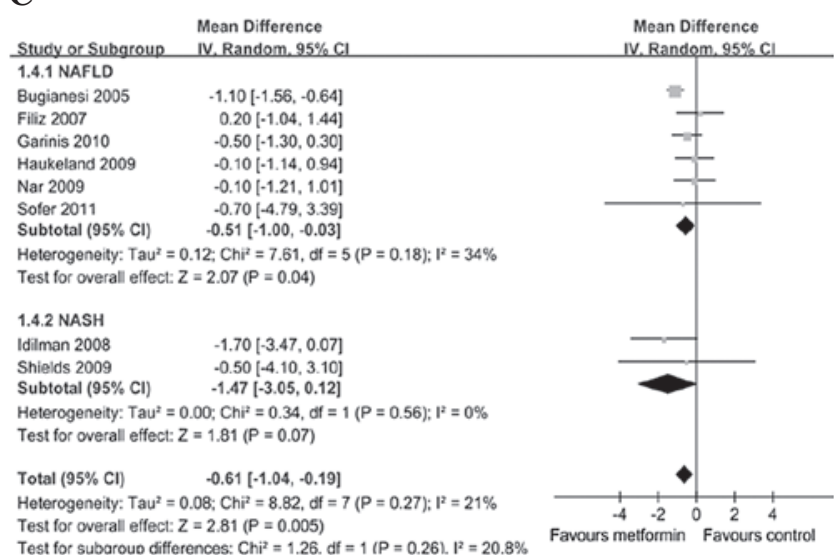

B

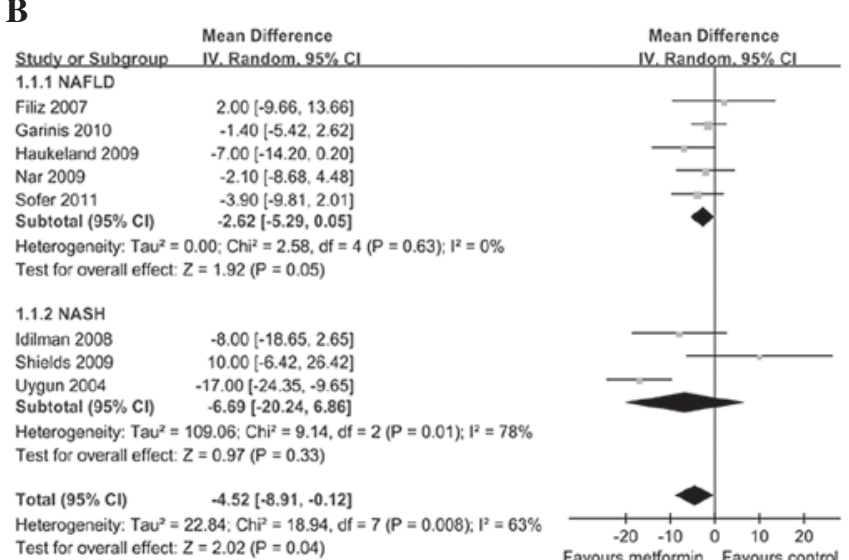

Heterogeneity: Tau $^{2}=22.84: C h i^{2}=18.94, d f=7(P=0.008): I^{2}=63 \%$
Test for overall effect: $Z=2.02(P=0.04)$

Favours metformin Favours control

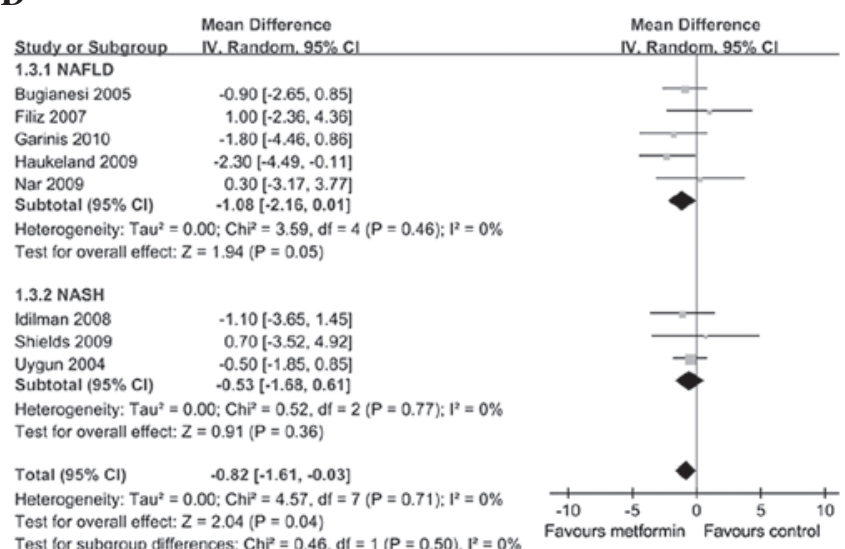

Figure 4. Forest plots of improvement in biochemical and anthropometric variables are shown. (A) Alanine aminotransferase (ALT); (B) aspartate aminotransferase (AST); (C) homeostasis model assessment of insulin resistance (HOMA-IR) and (D) body mass index (BMI).

in the metformin group $(20,22,25,28)$. In their study, Haukeland et al (20) reported that only one patient had developed exanthema. However, none of the patients discontinued metformin due to intolerance during treatment.

\section{Discussion}

In this systematic review, nine randomized clinical trials were included, six of which were on NAFLD and three on NASH. The nine trials had a small sample size and a high variability of group size (ranging from 19 to 82 participants). A total of 417 participants were enrolled. A number of patients were overweight or had diabetes. Two trials were considered to be of high methodological quality $(20,21)$, in terms of allocation sequence generation, allocation concealment, blinding and outcome data. The remaining seven trials (22-28) were inadequate or unclear in at least one of the five components used to assess methodological quality. Considerable heterogeneity, such as inclusion criteria, sample sizes, duration of treatment and methods of outcome assessment were present in these trials. Thus, the adequacy of this evidence should be considered carefully.

The effect of metformin on liver histology remains unclear. Our findings demonstrate that metformin does not improve the condition of NAFLD or NASH patients with the histological spectrum of steatosis, inflammation, hepatocel- lular ballooning and fibrosis, consistent with the findings of previously published systematic studies $(17,29,30)$. The current data should be interpreted objectively and dialectically. First, although the nine studies assessed the effect of metformin on histological response, five studies could not be included in the histological response analyses, due to the fact that three of them provided insufficient data and two employed US as the means of diagnosis. Furthermore, the histopathologic grading system was different in the four included studies, inevitably resulting in heterogeneity. The two studies assessing hepatic steatosis by US also suggested that metformin had no significant effect on hepatic steatosis. Although perfect evidence is absent, the studies included in this review demonstrate that metformin has no effect on histological response in NAFLD.

Regarding the liver enzymes, there were eight and nine studies assessing the effect of metformin on ALT and AST, respectively. Overall, they showed a statistically significant reduction in ALT and AST levels in the metformin group. Certain reviews $(31,32)$ have shown improvements in liver enzymes only in the single-arm trails. However, this has not yet been confirmed by data obtained from RCTs. Therefore, our findings may be more objective and reliable. In the NASH subgroup analysis, the metformin group had a tendency to exhibit lower ALT and AST levels compared to the control group, although this tendency was not statistically significant $(\mathrm{P}=0.62$ and 0.33$)$. A number of the included studies reported 
ALT to be a marker for hepatic inflammation. We found an average ALT reduction of $8.12 \mathrm{U} / 1$. However, ALT should not be considered to be a marker for liver improvement, since improvement in liver tests is not correlated with histological findings $(33,34)$.

IR was assessed using HOMA-IR (20-24,26-28) and serum insulin levels, in eight and one study, respectively (25). As expected, insulin sensitivity markedly improved in the metformin, compared to the control group. In their study, Rakoski et al (17) reported that non-diabetic patients may be particularly susceptible to the insulin-sensitizing properties of glitazone and that early intervention may prevent worsening of IR (35). However, our subgroup analysis detected no statistically significant differences between diabetic and nondiabetic patients, suggesting only that the improving trend in HOMA-IR was more obvious in NAFLD compared to NASH patients.

Due to the incomplete data on body weight, we calculated BMI instead and found a favourable response to metformin treatment in patients with NAFLD. This improvement in BMI was associated with an improvement in IR, as HOMA-IR scores were markedly lower at the end of the included studies in the two groups. However, three included studies showed no improvement in BMI $(21,26,28)$. The reason for this result is unclear, but may be associated with the relatively low dose in the three studies (mean dose of $0.76 \mathrm{~g} / \mathrm{day}$ ). Therefore, the dose or the duration of therapy should be more carefully determined in metformin treatment.

Our study had several limitations. First, the methodological limitations: the small number of patients, the lack of randomization and blinded measures and the incomplete histological outcomes. Second, although publication bias is a significant factor (assessed using funnel plots), it is uninformative due to the small number of studies available for our analysis. However, the included studies yielded negative results for an improvement in histological response. Third, meta-analysis of weighting studies draws conclusions resulting in the largest variance for the pooled effect size. Thus, 95\% CIs for the OR and MD are likely to be wider and more conservative than expected. We performed sensitivity analysis after excluding the studies that appeared as outliers in the forest plots, which did not have a significant impact on the results. Finally, the histopathologic grading system was inconsistent in the included studies, likely to have affected the accuracy of the data.

In the included studies, the duration of treatment ranged from 4 to 12 months (median duration, 8.5 months), and the dose of metformin ranged from 0.85 to $3 \mathrm{~g} /$ day (median dose, $1.5 \mathrm{~g} /$ day). Due to the limited number of the included studies, the optimal dose and the duration of therapy were difficult to determine. In addition, IR is widely considered to be a pivotal feature of NAFLD, which is strongly and independently associated with the increased risk of type 2 diabetes $(36,37)$. Although metformin has been used for the treatment of diabetes for $>50$ years, it has currently become one of the first-line treatment options in the management of diabetes, and is recommended by a number of international guidelines and consensus (38). Metformin may therefore be used to treat NAFLD and diabetes simultaneously. Therefore, application of the optimal dose of metformin in order to decrease NAFLD- associated risk of diabetes is a promising treatment approach. Further randomized clinical trails are required to confirm his hypothesis. This review shows that metformin has mild side-effects, thus can be used safely for long periods of time, and that no patient discontinued metformin due to intolerance or side-effects. However, we should be cautious about this conclusion, since evidence pointing towards important renal toxicity was reported in previous studies (39). The unexpected adverse events should be monitored carefully in future studies. Although we cannot determine the optimal dose and duration of therapy, the studies included in this review provide current available evidence on the beneficial as well as harmful effects of metformin in NAFLD.

Due to the heterogeneity of the inclusion criteria for patient enrollment, patients aged $<18$ years of age were excluded. However, several studies have evaluated the potential involvement of metformin in the therapy of pediatric patients with NAFLD (40-42). Findings regarding pediatric patient population were similar to those regarding adults, supporting the beneficial effects of metformin on biochemical and metabolic, but not on histological response. Despite the fact that this systematic review shows no histological benefit and a modest biochemical and metabolic benefit of metformin, whether or not metformin should be widely used in clinical trials remains to be determined. Investigators and clinicians should be aware of this issue, since the changes in histology in NAFLD have been observed in larger studies with long follow-up periods. In this systematic review, the follow-up period of the included trials on average was 8.5 months. Since the sample size was small, RCTs with large sample sizes and a long follow-up need to be conducted. The use of metformin might be limited to NAFLD to improve biochemical and metabolic features and might not be used for NASH. Furthermore, as metformin is less expensive compared to most other treatment modalities for NAFLD, it is considered affordable for the affected population.

In summary, this systematic review has shown that metformin may not improve histological response, but it can improve biochemical and metabolic features in NAFLD. Therefore, metformin remains a promising drug for the treatment of NAFLD due to its metabolic effects and safety profile. However, in the treatment of NASH, our subgroup analyses do not support this finding. RCTs with a low risk of bias need to be conducted in order to assess the beneficial or harmful effects of metformin on NAFLD or NASH. Moreover, RCTs with a large sample size and long follow-up period should be designed. Furthermore, future studies should develop a uniform assessment method of liver biopsy and investigate the optimal dose and duration of metformin therapy to achieve the maximal sustainable effect.

\section{Acknowledgements}

The authors would like to thank Ms. Xie-Wan Chen and Ms. Xiao-Qing Zhan (Medical English Department, Third Military Medical University, Chongqing, China) for the critical reading of the manuscript and their valuable advice. This study was supported by the Natural Science Foundation of China (grant no. 81072362 and 81200297). 


\section{References}

1. Cohen JC, Horton JD and Hobbs HH: Human fatty liver disease: old questions and new insights. Science 332: 1519-1523, 2011.

2. Chalasani N, Younossi Z, Lavine JE, et al: The diagnosis and management of non-alcoholic fatty liver disease: practice guideline by the American Gastroenterological Association, American Association for the Study of Liver Diseases, and American College of Gastroenterology. Gastroenterology 142: $1592-1609,2012$

3. Wieckowska A, McCullough AJ and Feldstein AE: Noninvasive diagnosis and monitoring of nonalcoholic steatohepatitis: present and future. Hepatology 46: 582-589, 2007.

4. Liou I and Kowdley KV: Natural history of nonalcoholic steatohepatitis. J Clin Gastroenterol 40: S11-S16, 2006.

5. Farrell GC and Larter CZ: Nonalcoholic fatty liver disease: from steatosis to cirrhosis. Hepatology 43: S99-S112, 2006.

6. Alberti KG, Zimmet P and Shaw J; IDF Epidemiology Task Force Consensus Group: The metabolic syndrome - a new worldwide definition. Lancet 366: 1059-1062, 2005.

7. Targher G, Day CP and Bonora E: Risk of cardiovascular disease in patients with nonalcoholic fatty liver disease. $\mathrm{N}$ Engl J Med 363: 1341-1350, 2010

8. Dunn W, Xu R, Wingard DL, et al: Suspected nonalcoholic fatty liver disease and mortality risk in a population-based cohort study. Am J Gastroenterol 103: 2263-2271, 2008.

9. Angulo P: Nonalcoholic fatty liver disease. N Engl J Med 346: 1221-1231, 2002.

10. McCullough AJ: Update on nonalcoholic fatty liver disease. J Clin Gastroenterol 34: 255-262, 2002.

11. Owen MR, Doran E and Halestrap AP: Evidence that metformin exerts its anti-diabetic effects through inhibition of complex 1 of the mitochondrial respiratory chain. Biochem J 348: 607-614, 2000.

12. Zhou G, Myers R, Li Y, et al: Role of AMP-activated protein kinase in mechanism of metformin action. J Clin Invest 108 $1167-1174,2001$

13. Browning JD and Horton JD: Molecular mediators of hepatic steatosis and liver injury. J Clin Invest 114: 147-152, 2004.

14. Moseley RH: Therapy for nonalcoholic fatty liver disease. J Clin Gastroenterol 42: 332-335, 2008.

15. Loomba R, Lutchman G, Kleiner DE, et al: Clinical trial: pilot study of metformin for the treatment of non-alcoholic steatohepatitis. Aliment Pharmacol Ther 29: 172-182, 2009.

16. Lam B and Younossi ZM: Treatment options for nonalcoholic fatty liver disease. Therap Adv Gastroenterol 3: 121-137, 2010.

17. Rakoski MO, Singal AG, Rogers MA and Conjeevaram $\mathrm{H}$ : Meta-analysis: insulin sensitizers for the treatment of non-alcoholic steatohepatitis. Aliment Pharmacol Ther 32: $1211-1221,2010$

18. Mazza A, Fruci B, Garinis GA, et al: The role of metformin in the management of NAFLD. Exp Diabetes Res 2012: 716404 2012.

19. Higgins PTJ and Green S: Cochrane Handbook for Systematic Reviews of Interventions. Version 5.1.6. The Cochrane Collaboration, 2011 (Available at: www.cochrane-handbook. org). Accessed April 2, 2012.

20. Haukeland JW, Konopski Z, Eggesbo HB, et al: Metformin in patients with non-alcoholic fatty liver disease: a randomized, controlled trial. Scand J Gastroenterol 44: 853-860, 2009.

21. Shields WW, Thompson KE, Grice GA, et al: The effect of metformin and standard therapy versus standard therapy alone in nondiabetic patients with insulin resistance and nonalcoholic steatohepatitis (NASH): a pilot trial. Therap Adv Gastroenterol 2: 157-163, 2009

22. Sofer E, Boaz M, Matas Z, et al: Treatment with insulin sensitizer metformin improves arterial properties, metabolic parameters, and liver function in patients with nonalcoholic fatty liver disease: a randomized, placebo-controlled trial. Metabolism 60: $1278-1284,2011$.
23. Bugianesi E, Gentilcore E, Manini R, et al: A randomized controlled trial of metformin versus vitamin $\mathrm{E}$ or prescriptive diet in nonalcoholic fatty liver disease. Am J Gastroenterol 100: 1082-1090, 2005

24. Garinis GA, Fruci B, Mazza A, et al: Metformin versus dietary treatment in nonalcoholic hepatic steatosis: a randomized study. Int J Obes (Lond) 34: 1255-1264, 2010.

25. Uygun A, Kadayifci A, Isik AT, et al: Metformin in the treatment of patients with non-alcoholic steatohepatitis. Aliment Pharmacol Ther 19: 537-544, 2004

26. Akyüz F, Demir K, Ozdil S, et al: The effects of rosiglitazone, metformin, and diet with exercise in nonalcoholic fatty liver disease. Dig Dis Sci 52: 2359-2367, 2007.

27. Idilman R, Mizrak D, Corapcioglu D, et al: Clinical trial: insulin-sensitizing agents may reduce consequences of insulin resistance in individuals with non-alcoholic steatohepatitis. Aliment Pharmacol Ther 28: 200-208, 2008.

28. Nar A and Gedik O: The effect of metformin on leptin in obese patients with type 2 diabetes mellitus and nonalcoholic fatty liver disease. Acta Diabetol 46: 113-118, 2009.

29. Musso G, Gambino R, Cassader M, et al: A meta-analysis of randomized trials for the treatment of nonalcoholic fatty liver disease. Hepatology 52: 79-104, 2010.

30. Musso G, Cassader M, Rosina F and Gambino R: Impact of current treatments on liver disease, glucose metabolism and cardiovascular risk in non-alcoholic fatty liver disease (NAFLD): a systematic review and meta-analysis of randomised trials. Diabetologia 55: 885-904, 2012.

31. Chavez-Tapia NC, Barrientos-Gutierrez T, Tellez-Avila FI, et al: Insulin sensitizers in treatment of nonalcoholic fatty liver disease: Systematic review. World J Gastroenterol 12: 7826-7831, 2006.

32. Adams LA and Angulo P: Treatment of non-alcoholic fatty liver disease. Postgrad Med J 82: 315-322, 2006

33. Mofrad P, Contos MJ, Haque M, et al: Clinical and histologic spectrum of nonalcoholic fatty liver disease associated with normal ALT values. Hepatology 37: 1286-1292, 2003.

34. Adams LA, Sanderson S, Lindor KD, et al: The histological course of nonalcoholic fatty liver disease: a longitudinal study of 103 patients with sequential liver biopsies. J Hepatol 42: 132-138, 2005.

35. Orlando R, Azzalini L, Orando S, et al: Bile acids for non-alcoholic fatty liver disease and/or steatohepatitis. Cochrane Database Syst Rev 1: CD005160, 2007.

36. Filik L: Role of type 2 diabetes mellitus in nonalcoholic fatty liver disease. Eur J Clin Invest 41: 1367-1368, 2011.

37. Bonapace S, Perseghin G, Molon G, et al: Nonalcoholic fatty liver disease is associated with left ventricular diastolic dysfunction in patients with type 2 diabetes. Diabetes Care 35: 389-395, 2012.

38. Stumvoll M, Nurjhan N, Perriello G, et al: Metabolic effects of metformin in non-insulin-dependent diabetes mellitus. N Engl J Med 333: 550-554, 1995.

39. Hemmingsen B, Christensen LL, Wetterslev J, et al: Comparison of metformin and insulin versus insulin alone for type 2 diabetes: systematic review of randomised clinical trials with meta-analyses and trial sequential analyses. BMJ 344: 1771-1779, 2012.

40. Nadeau KJ, Ehlers LB, Zeitler PS, et al: Treatment of nonalcoholic fatty liver disease with metformin versus lifestyle intervention in insulin-resistant adolescents. Pediatr Diabetes 10: $5-13,2009$.

41. Lavine JE, Schwimmer JB, Van Natta ML, et al: Effect of vitamin $\mathrm{E}$ or metformin for treatment of nonalcoholic fatty liver disease in children and adolescents: the TONIC randomized controlled trial. JAMA 305: 1659-1668, 2011.

42. Lavine JE, Schwimmer JB, Molleston JP, et al: Treatment of nonalcoholic fatty liver disease in children: TONIC trial design. Contemp Clin Trials 31: 62-70, 2010. 